\title{
La cenere e la lacrima. Per raccontare il massacro degli ebrei di Kiev
}

\author{
Stefano Petrungaro*
}

\section{Ashes and tears. Narrating the massacre of Kiev's Jews}

Antonella Salomoni investigates the memory of the massacre of Kiev's Jews, which took place between the $29^{\text {th }}-30^{\text {th }}$ of September 1941, and which was perpetrated by the Nazi occupying forces, supported by local Ukrainian collaborators. The official and public discourse during the Soviet era put the emphasis on the common heroic struggle of the Soviet people against the Nazi aggression, silencing or even negating the actual anti-Semitic nature of the mass-killing. The study provides a sophisticated examination of the artistic voices which took shape in the field of poetry, music and painting, and which undertook a struggle with Soviet authorities in order to remember those facts. Notwithstanding the great emotive and professional difficulties they had to suffer, these authors fought against the official efforts at removing - even physically - the traces and memory of the massacre.

Key words: Holocaust, Memory, Soviet Union, Ukraine

Parole chiave: Shoah, Memoria, Unione Sovietica, Ucraina

Scriveva nel 1947 il poeta Jakov Chelemskij, citato in apertura del volume di Antonella Salomoni, che chi avesse dimenticato la tragedia avvenuta a Babij Jar, nel ricordarla sarebbe stato investito improvvisamente da «una desolante nube di polvere e cenere» ${ }^{1}$. Il volume qui recensito riesce, con rigore e grande sensibilità, a guidare il lettore attraverso quella nube, restituendo l'intrico di attori e fattori che hanno determinato l'eccidio prima, il suo oblio ufficiale poi. Lo studio non è tanto una storia del massacro, quanto della tensione sorta tra le politiche della memoria sovietiche, che per quei fatti avevano decretato l'oblio, e le resistenze opposte da chi invece si sforzò di dargli voce.

L'evento in sé viene comunque dettagliatamente ricostruito nelle pagine di un'intensa introduzione che si snoda attraverso una sessantina di pagine. Lì si

* Dslcc, Dorsoduro 1405, 30123 Venezia; stefano.petrungaro@unive.it

${ }^{1}$ J. Chelemskij, Nikogda v etom gorode ne bylo pyli (1946), cit. in A. Salomoni, Le ceneri di Babij Jar. L'eccidio degli ebrei di Kiev, il Mulino, Bologna 2019, p. 8. Altre esecuzioni erano iniziate già nei giorni precedenti e molte altre, con modalità assai differenti e vittime non solo ebraiche, proseguiranno nei mesi successivi, sempre nella stessa area: ivi, pp. 38-39. 
narrano le modalità attraverso le quali le truppe tedesche, che avevano occupato l'Ucraina, sterminarono a Kiev tra il 29 e il 30 settembre, a colpi d'arma da fuoco, 33.771 ebrei, come riporta il primo rapporto ufficiale (p. 35). L'eccidio avvenne non in un luogo lontano dalla capitale e dagli sguardi della popolazione, bensì ai margini della città, in quell'enorme «burrone» (jar) chiamato «Babij Jar». Un comunicato diffuso il 28 settembre imponeva a tutti gli ebrei di Kiev e dintorni di presentarsi il giorno successivo, portando con sé documenti, denaro, vestiti pesanti e biancheria. Ignari delle politiche antisemite del nazionalsocialismo, anche in virtù del patto Molotov-Ribbentropp, la popolazione ebraica della capitale ucraina si presentò alla convocazione, in buona parte convinta di andare incontro a un reinsediamento. Prese così forma, come riportano le testimonianze, un «immenso torrente» (p. 23) di persone, una «processione di morte [che] durò tre giorni e tre notti» (p. 25), sotto gli occhi dei concittadini ucraini. Alla meticolosa spoliazione delle vittime, accompagnata da insulti e angherie, seguiva l'uccisione con un colpo alla nuca, non prima di aver disposto le vittime in modo da sfruttare al massimo lo spazio del burrone. Del massacro, coadiuvato dalle forze collaborazioniste ucraine, si seppe subito, in città e all'estero.

Fin dall'inizio, la riflessione è costruita con un deciso impianto multiprospettico, che è insieme elemento stilistico e metodologico, formale e di contenuto: la rievocazione ripetuta e insistita dello stesso evento narrato da diversi attori permette anzitutto di coglierne aspetti differenti, derivanti dai distinti punti di vista, e di avere un quadro generale complesso e anche scomposto. L'Autrice non nasconde infatti le discrasie e le parzialità delle testimonianze, piuttosto discutendole, ad esempio all'inizio, nel descrivere la marcia delle vittime verso il luogo dell'esecuzione, come pure alla fine, nelle diverse descrizioni delle manifestazioni, a seconda che venissero rese dai partecipanti o dagli agenti di sicurezza. Il testo viene così a essere un elegante laboratorio, dove il metodo storico è toccato con mano.

Tenuto conto però del tema, non è solo un esercizio di metodo. Si tratta anche di esibire la base documentaria di una catena di fatti che, fin da subito, è stata ed è oggetto di contestazione e persino negazionismo. Sebbene la autorità sovietiche avessero una conoscenza particolareggiata dell'eccidio fin dal dicembre 1941, già subito dopo la liberazione della città, il 6 novembre 1943, iniziò la deformazione e negazione ufficiale degli avvenimenti (pp. 6167). Secondo un modello interpretativo che influenzò il discorso pubblico e la storiografia per tutto il periodo sovietico, il massacro doveva essere privato del suo carattere antiebraico e subordinato al racconto della "Grande guerra patriottica". Secondo tale impostazione, l'aggressione era stata rivolta contro l'intero paese e non una sua sola componente, e le vittime andavano intese come «pacifici cittadini sovietici», trascurandone l'identità ebraica e misconoscedo così la vera natura del massacro. Ciò si lega ovviamente anche al diffuso antisemitismo, che non tardò a (ri)manifestarsi in più forme persino nella Kiev appena liberata, anche attraverso un ridimensionamento o addirittura una negazione delle politiche persecutorie naziste (pp. 98-106). 
La riproposizione, sempre predisposta accuratamente e inserita in un quadro analitico, delle evidenze documentarie serve quindi anche a difendere la storicità di quei fatti, invitando così con fermezza a non distogliere lo sguardo dalle fiamme «in cui si dimenava il Babij Jar»², piuttosto raccogliendone e tematizzandone le ceneri. Le ceneri di Babij Jar sono, all'inizio, quelle dei corpi riesumati e bruciati nell'estate del 1943 da una speciale unità, guidata dalle SS ma composta da detenuti del vicino campo di concentramento di Syrec', secondo quella riterritorializzazione dei massacri che caratterizza tutta l'Europa orientale e che mirava alla cancellazione delle tracce dei crimini di guerra nazisti. I resti dei cadaveri dissotterrati furono così arsi su enormi pire, che bruciarono per giorni. Circa settantamila corpi vennero così inceneriti, e quelle ceneri vennero poi obbligatoriamente disperse per il burrone.

$\mathrm{Si}$ intravede subito la valenza polisemantica del titolo Le ceneri di Babij Jar. La cenere può infatti classicamente essere associata alla rimanenza, a quel che resta dopo il fuoco. Un motivo assai esplorato, nelle arti come nelle scienze umane, quello della cenere in quanto resto, sia di ciò che «fu» $(\mathrm{feu})$, sia di ciò che è stato dato al «fuoco» $(f e u)$, e ancora dalle numerose implicazioni teoretiche ${ }^{3}$. La cenere come traccia, come metafora di un evento che si è consumato: qui, un evento tragico, che richiede riconoscimento, quindi ricordo e rispetto. La cenere su cui si basa il ricordo di Babij Jar, quindi, non è solo una metafora. Il testo lo ricorda in più punti, come quando l'architetto A.F. Ignaščenko narra che, nel corso dei lavori per gettare le fondamenta del monumento eretto nel 1976, fu rinvenuto uno spesso strato di cenere umana ${ }^{4}$. E ancora prima Anatolij V. Kuznecov, in occasione delle perlustrazioni in vista della preparazione del suo noto romanzo-documento ${ }^{5}$, notò bambini che sminuzzavano e setacciavano dei blocchi di carbone e cenere compatta, alla ricerca di orecchini, denti, anelli: «Era la cenere di molte persone, in cui tutto si era rimescolato; una sorta di cenere internazionale» ${ }^{6}$. L'internazionalità attribuita alla cenere rimanda all'approccio di Kuznecov che, pur sottolineando il carattere antisemita dell'eccidio, inquadrava la distruzione del popolo ebraico all'interno di «una tragedia umana collettiva». Era questo uno snodo interpretativo cruciale perché, come ricordato, attorno all'assegnazione alle vittime di un'identità ebraica, o alla loro definizione, secondo l'ottica ufficiale, più generale di «cittadini sovietici», si giocava l'approvazione della censura.

Anche l'opera di Kuznecov, come tutta la produzione del periodo sovietico su Babij Jar, fu sottoposta a un degradante trattamento di revisione, tagli e riscrittura. Alle difficoltà, le sofferenze, talvolta gli squilibri emotivi provati da

${ }^{2}$ Dal poema Jar di Mykola P. Bažan (1943), ivi, pp. 58-59.

${ }^{3}$ J. Derrida, Ciò che resta del fuoco, SE, Milano 2000 (ed. or. 1999).

${ }^{4}$ Pur non essendo il luogo dove erano avvenute le fucilazioni: A. Salomoni, Babij Jar cit., p. 316, n. 122 .

${ }^{5}$ A. Kuznecov, Babij Jar. Romanzo-documento, Adelphi, Milano 2019, apparso a puntate nel 1966 e in versione integrale nel 1970.

${ }^{6}$ Cit. in A. Salomoni, Babij Jar cit., p. 225. 
questi autori già solo per il fatto di occuparsi di una simile vicenda, si dovevano aggiungere, come è ben documentato nel caso di Evgenij A. Evtušenko, di Viktor P. Nekrasov e di Kuznecov stesso, i tormenti indotti dalle autorità nei confronti di artisti ai quali era ostacolata e a volte del tutto impedita l'attività creativa e professionale. È così che, come disse Nekrasov, egli come scrittore, «vale a dire come uomo che non solo scrive, ma che anche pubblica i propri scritti», da un certo momento in poi cessò «di esistere» ${ }^{7}$.

Nonostante questa immensa fatica, più d'una voce artistica riuscì a prender forma. Nel silenzio ufficiale che tentava di promuovere l'oblio di Babij Jar, individui coraggiosi decisero di nominare la tragedia e di metterla in scena. Questi gesti intellettuali sembrano così rinviare alla ulteriore valenza della figura della cenere, che può essere non solo ciò che non è più, ma anche ciò che non è ancora. È traccia, fragile segno di ciò che è stato, ma può essere raccolta per scrivere. Come per il nerofumo degli antichi, ossia la miscela di cenere e acqua da cui nacque il primo inchiostro, la cenere si intreccia alla scrittura. Anche nel caso di Babij Jar, il ricordo e il silenzio furono così tramutati in parola, letta declamata e cantata, in immagini e note musicali. Il volume di Salomoni diventa così un'accurata e affascinante guida attraverso la creatività artistica, la sua forza di denuncia, il suo ruolo cruciale in Ucraina e Unione sovietica nel trattenere, innovare e attualizzare la vicenda del massacro degli ebrei kieviani. Le ceneri di Babij Jar vennero così sottratte al destino che era stato loro previsto, per farsi invece voce, sussurro e talvolta grido. La cenere non è più l'ombra di un'assenza, ma evoca la presenza: sancisce la perdita irrimediabile, ma al contempo ne commemora il ricordo e lo attualizza. Le fiamme nel burrone, quindi, hanno effettivamente dato luogo inizialmente a una dispersione, ma poi anche a una disseminazione.

Già nel settembre 1944, Itzik Kipnis rivolgeva un accorato invito: «Ebrei, cari fratelli, solleviamoci da terra, scrolliamoci di dosso le ceneri e facciamo risplendere tutta la luce che il nostro popolo porta con sé» (p. 125). Quella di Kipnis, di cui si vuole liberare, è la cenere del silenzio, che le autorità vorrebbero stendere sull'operazione antisemita e in generale sull'antisemitismo della società russa. Ma «A volte anche il silenzio equivale alla menzogna», risponde Evegenij A. Evtušenko, durante un incontro ufficiale, direttamente a Chruščëv (p. 203). C'era quindi un vuoto di parole nella retorica ufficiale, piena del nuovo canone narrativo della Grande guerra patriottica, che sottolineava la riconciliazione nazionale e non lasciava spazio a conflitti interni e vittimismi, soprattutto se le vittime in questione erano ebree. A quel vuoto delle parole e della memoria collettiva si associava quindi il drammatico vuoto di una comunità nella comunità locale, venuta meno. Un vuoto che si rifletteva nell'assenza di qualunque monumento o targa sul luogo del massacro, dove comunque avevano luogo iniziative private di commemorazione. Un vuoto che, come l'intero burrone di Babij Jar, si decise di eliminare. Dopo anni di abbandono dell'intera area, trasformatasi in una discarica, si tentò di «colma-

${ }^{7}$ Cit. ivi, p. 311. 
re» la voragine, di «coprire» Babij Jar e «pareggiare» il terreno (p. 159), scaricandovi scarti di fabbricazione di materiale edilizio, mescolati con acqua. Si decise quindi di fare del vuoto un pieno, riempiendo quello spazio col fango, fatto di ceneri, terra, acqua e lacrime - queste ultime qui ancora nascoste.

Anche prima della guerra Šostakovič ricorda che le lacrime andavano piante in silenzio: ognuno era solo con la propria pena, e il dolore andava soffocato. Con lo scoppio della guerra, invece, «potevamo piangere apertamente [...] La gente non ebbe più paura delle lacrime» (p. 195). Perché le lacrime possono essere pericolose, quando rivelano un'ingiustizia o una discriminazione. Possono quindi essere oggetto persino di divieti: vietato piangere. Le lacrime, poi, intaccano il modello virilista di un paese in armi: gli uomini tutti d'un pezzo non possono piangere, almeno non in pubblico, e nemmeno le nazioni vincenti devono piangersi addosso, bensì celebrare le proprie virtù, soprattutto guerriere, e questo fu quanto avvenne in Unione Sovietica, come in numerosi altri paesi europei, dopo il secondo conflitto mondiale.

Ma vi sono momenti in cui le lacrime sono legittime e accettate. Nel libro a un certo punto si inizia a piangere insieme, in pubblico, attraverso riti collettivi di denuncia e liberazione. Si leggono descrizioni di scene toccanti, come quelle descritte da Evtušenko, che nell'autunno del 1961 dedica un suo poema al massacro degli ebrei di Kiev. In un paese dove la lettura e la poesia svolgono un ruolo importante, forse più che altrove (p. 85), accade che i poeti leggano a voce alta i propri versi davanti a pubblici numerosi, che ascoltano commossi. Quindi le lacrime iniziano a scorrere e ad essere condivise, in sale stipate ma in religioso silenzio, dove i presenti ascoltano in tensione, finché la tensione si fa dolore e il dolore si scioglie in lacrima. L'emozione di sentire che certe parole, finalmente, vengono pronunciate.

L'arte, anzitutto la poesia e la musica (e poesia in musica, ché di opere cantate spesso si tratta, come nella sinfonia n. 13 di Šostakovič, su un testo di Evtušenko), come necessità di vincere la paura che, già all'epoca dello stalinismo, aveva avuto il sopravvento sulla parola (p. 203). A fronte del silenzio, o del disprezzo esibito dalla critica, le sale dei teatri si riempiono di applausi, i testi censurati continuano a circolare illegalmente. È per mezzo dei versi, racconta Salomoni, che Babij Jar continua a essere presente nelle società ucraina e russa. I versi sorreggono quindi questo volume, un magistrale lavoro di fine sartoria, che abilmente cuce le citazioni nel testo e nei titoli: c'è un profondo rispetto da parte dell'autrice nei confronti della parola, e di queste parole, al punto da fare spesso un passo indietro, e lasciare il campo alla voce di poeti e scrittori, che ci parlano di scene, sentimenti, come pure di odori e suoni.

Nonostante la commozione che quelle parole suscitano, non è una storia lacrimosa quella ricostruita da Salomoni. Sono sì lacrime di sofferenza ma anche di rabbia, di determinazione. Gli attori coinvolti nel mantenimento, o meglio nel recupero della memoria di Babij Jar, combattono con incredibile fermezza le politiche censorie sovietiche. C'è chi ha il coraggio di contraddire in faccia Chruščëv, chi tiene testa alle critiche negative dei media, chi ingaggia 
duelli, sebbene da posizioni profondamente asimmetriche, con le commissioni di partito per l'approvazione dei testi da pubblicare. Vi sono dialoghi riportati nel testo che lasciano senza fiato, per la lucidità e la determinazione con cui gli attivisti rispondono alle intimidazioni della polizia (es. pp. 300-01).

La storia narrata da Salomoni è così la vicenda tormentata dell'interazione tra numerosi attori: lungi dall'esser solo la ricostruzione delle politiche della memoria sovietiche riguardanti la Shoah, di cui l'Autrice si è già approfonditamente occupata ${ }^{8}$, questo lavoro dà forma via via a un'indagine delle pratiche concrete e delle relazioni tra artisti e intellettuali, gli editori, gli apparati del partito, la società. È dalla negoziazione di ogni pubblicazione, esecuzione, lettura, indagine sul campo, che prende corpo quel multiforme processo qui accuratamente riassemblato. Un processo così lungo e complesso non può che avere ricadute anche assai diverse. Nel penultimo e ancor più nell'ultimo capitolo viene illustrato come, a partire dagli anni '60 e soprattutto negli anni '70, la commemorazione dell'eccidio incontrò istanze di vario tipo, come la denuncia dell'«ingiustizia» subita dagli ucraini ad opera dei sovietici (leggi: carestia del 1932-33 e repressione delle istanze nazionali), associata se non equiparata a quella subita dagli ebrei; la militanza di certa intelligencija russa per i diritti umani e civili; la lotta contro l'antisemitismo; la militanza sionista; le battaglie per il diritto a emigrare in Israele.

Il volume accenna nelle ultime righe agli sviluppi successivi all'ottenimento dell'indipendenza dell'Ucraina nel 1991. Si aprì una nuova fase, caratterizzata dal riconoscimento ufficiale di Babij Jar quale eccidio antisemita, ma anche da pluralità e conflitti di memorie tipicamente post-sovietici, che nel caso specifico si radicano nei contrasti ucraino-russi e nel difficile rapporto tra nazionalismo ucraino e minoranze locali. Da qui potrebbe ripartire l'indagine, inserendo gli sviluppi delle dinamiche memoriali locali all'interno di quelli dell'Ucraina post-socialista e del suo rapporto con la memoria della Shoah, in chiave comparata rispetto alla Federazione russa, come a tutta l'Europa centro- e sud-orientale. Sono infatti sempre più numerosi gli studi che affrontano la frammentazione delle memorie post-socialiste, la nazionalizzazione delle memorie collettive, la contrapposizione tra esperienza del Gulag e la Shoah'.

Ma non erano evidentemente questi i temi che interessavano all'autrice, che si è piuttosto dedicata a ricostruire un doloroso capitolo di storia sovietica. Anche in relazione a questo periodo il caso di studio ucraino e sovietico potrebbe essere proficuamente messo in dialogo con altre esperienze socialiste est-europee. Studi recenti integrano l'immagine di un Blocco socialista nel quale alla perse-

${ }^{8}$ A. Salomoni, L'Unione Sovietica e la Shoah. Genocidio, resistenza, rimozione, il Mulino, Bologna 2007.

${ }_{9}^{9}$ J.-P. Himka-J.B. Michlic (eds.), Bringing the Dark to Light: The Reception of the Holocaust in Postcommunist Europe, Nebraska UP, Lincoln 2013; M. Pakier-J. Wawrzyniak (eds.), Memory and Change in Europe: Eastern Perspectives, Berghahn Books, New York-Oxford 2016; N. Ragaru (dir.), La Shoah en Europe du Sud-Est. Les Juifs en Bulgarie et dans les territoires sous administration bulgare (1941-1944), E-Editions du Mémorial de la Shoah, Paris 2014; Aa.Vv., War and Memory in Russia, Ukraine and Belarus, Palgrave Macmillan, London 2017. 
cuzione degli ebrei non veniva data un'adeguata attenzione pubblica e scientifi$\mathrm{Ca}^{10}$, con approfondimenti sul mantenimento di memorie private, informali; ma anche su iniziative giudiziarie, archivistiche e di studio inter- e transnazionali, che hanno preso corpo in Europa orientale e hanno anche attraversato la presunta "cortina di ferro", ottenendo così un'immagine d'insieme più sfumata e composita del rapporto tra società socialiste e Shoah ${ }^{11}$. Ma alla dimensione comparata qui si predilige lo studio di caso in profondità, servendosi dell'ampia mole di studi specialistici, di fonti edite e inedite, al fine di ricostruire attentamente le vicende di singole personalità, (non-)monumenti, di luoghi e parole.

Se il confronto con la letteratura scientifica internazionale è serrato, ad occupare il centro del lavoro sono gli artisti e gli attivisti, di cui si studiano memorie e corrispondenze private, e le opere artistiche, delle quali si ricostruisce la genesi, si comparano le edizioni e traduzioni. È un approccio in parte necessario. Perché, come ricordato, è anzitutto attraverso il linguaggio artistico che si levano voci di indignata protesta nei confronti del destino di Babij Jar. L'Autrice non poteva non basare le sue riflessioni su poesie, opere sinfoniche e pittoriche. Ma c'è qualcosa di più in questa scelta, che non deriva dalla disponibilità delle fonti, avendo piuttosto una valenza metodologica più profonda. Sebbene il volume sia chiaramente una riflessione sul linguaggio e la sua manipolazione del linguaggio, sulla persistenza di vecchi "codici" antisemiti e sull'invenzione di nuove "parole" per nascondere e negare, la metodologia è intenzionalmente sotto traccia. Tuttavia, compare un riferimento, in nota, fugacemente presentato alla fine del libro, ai lavori di Martha C. Nussbaum, le cui riflessioni hanno «guidat[o]» l'Autrice, e che conferma l'importanza conferita al lavorìo artistico e alle sue implicazioni sociali' ${ }^{12}$. In quest'ottica e come già nell'antichità, le opere artistiche, nell'affrontare temi cruciali per la comunità quali l'ingiustizia, nonché sentimenti quali il dolore e la sofferenza, svolgono una importante funzione educativa e sociale. Il linguaggio e le pratiche artistiche, così come i libri che studiano quei fenomeni, hanno quindi un sostanziale afflato militante e politico in senso lato. Anche in epoca contemporanea, si sostiene, il fine delle arti e della riflessione critica è la promozione di una società liberale più giusta e libera, capace di compassione e rispetto per il prossimo. È anche in tal senso che l'intensa riflessione polifonica di Salomoni fornisce un importante contributo.

${ }^{10} \mathrm{Cfr}$ ad es. J.B. Michlic, Il passato e il futuro della memoria dell'Olocausto nell'Europa orientale: il caso della Polonia, in Aa.Vv., Storia della Shoah. La crisi dell'Europa, lo sterminio degli ebrei e la memoria del XX secolo, v. IV, UTET, Milano 2006, pp. 523-59; C. Tonini, Operazione Madagascar. La questione ebraica in Polonia 1918-1968, Clueb, Bologna 1999.

${ }^{11}$ Cfr. ad es. N. Ragaru, Bordering the Past: The Elusive Presences of the Holocaust in Socialist Macedonia and Socialist Bulgaria, «Südost-Forschungen», 76 (2017), pp. 241-72; Id., «Et les Juifs bulgares furent sauvés...» Une histoire des savoirs sur la Shoah en Bulgarie, Presses de Sciences Po, Paris 2020.

${ }_{12}$ M.C. Nussbaum, Giustizia poetica. Immaginazione letteraria e vita civile, Mimesis, Milano-Udine 2012; Ead., Emozioni politiche. Perché l'amore conta per la giustizia, il Mulino, Bologna 2014. 